\title{
Downregulation of ERBB3 decreases the proliferation, migration and invasion of cervical cancer cells though the interaction with MTK-1
}

\author{
JINGYUN DU, SHIHUA ZHOU, LI WANG, MULAN YU and LIYAN MEI \\ Department of Gynaecology and Obstetrics, Luodian Hospital, Shanghai 201908, P.R. China
}

Received October 9, 2017; Accepted March 28, 2018

DOI: $10.3892 / \mathrm{ol} .2018 .9088$

\begin{abstract}
Cervical cancer is a common malignancy in females. Diagnosis and treatment of cervical cancer remains a challenge due to difficulties in the presence of tumor metastasis. Increased expression level of Erb-b2 receptor tyrosine kinase 3 (ERBB3) has previously been demonstrated to be associated with the occurrence of cervical cancer; however, the functionality of ERBB3 in the development of cervical cancer remains incompletely understood. In the present study, the expression level of ERBB3 in patients with cervical squamous cell carcinoma and cervical adenocarcinoma was detected by reverse transcription quantitative polymerase chain reaction. The effects of ERBB3 small interfering RNA silencing on cell proliferation, migration and invasion were explored, and the interaction between ERBB3 and mitogen-activated protein kinase kinase kinase 4 (MTK-1) was also investigated. It was identified that the downregulation of ERBB3 significantly decreased the proliferative, migratory and invasive abilities of cervical cancer cells. In addition, the expression level of MTK-1 was also significantly decreased following MTK-1 siRNA silencing. Therefore, we hypothesize that the downregulation of ERBB3 may decrease the proliferative, migratory and invasive abilities of cervical cancer cells by inhibiting the expression of MTK-1.
\end{abstract}

\section{Introduction}

As the fourth most common type of malignant tumor in females, cervical cancer causes an unacceptably high mortality rate worldwide (1). Cervical cancer may be divided into two major subgroups, including cervical squamous cell carcinoma and cervical adenocarcinoma (2). As the dominant cervical

Correspondence to: Dr Jingyun Du, Department of Gynaecology and Obstetrics, Luodian Hospital, 121 Luoxilu, Baoshan, Shanghai 201908, P.R. China

E-mail:wnj1738558@gmail.com

Key words: cervical cancer, cervical squamous cell carcinoma, cervical adenocarcinoma, Erb-b2 receptor tyrosine kinase 3, mitogen-activated protein kinase kinase kinase 4 cancer type, cervical squamous cell carcinoma accounts for $80-90 \%$ of all cervical cancer cases (3). Human papillomaviruses (HPV) infection has been demonstrated to be closely associated with the occurrence and development of cervical cancer $(3,4)$, and associations between certain HPV genotypes and the incidence of cervical cancer are well-established (5). With the development of HPV infection screening programs and a continually increasing HPV vaccination rate, the incidence of cervical squamous cell carcinoma has been significantly reduced (3-5). However, the incidence of cervical adenocarcinoma has been demonstrated to have increased from 5 to $24 \%$ in the previous 30 years $(6,7)$, due to cervical cancer also being caused by factors other than HPV infection, and the prognosis of HPV-negative cervical cancer is usually poor (7). Therefore, the development of novel prevention and treatment modalities for cervical cancer are required.

The development of cervical cancer is a complex process with various internal and external factors involved (8). A recent study suggested that Erb-b2 receptor tyrosine kinase 3 (ERBB3) is likely to be involved in the development of cervical cancer (8). However, the functionality of ERBB3 in the pathogenesis of this disease remains unclear. A previous study indicated that ERBB3 may promote the migration and invasion of breast cancer cells and increased resistance of cancer cells to targeted therapy (9). In contrast, degradation of ERBB3 mediated by E3 ubiquitin-protein ligase NRDP1 (NRDP1) was demonstrated to inhibit the migration and invasion of human glioma cells (10). In light of the data from previous studies, it is reasonable to hypothesize that ERBB3 may also participate in the progression of cervical cancer by regulating the migration and invasion of cancer cells.

In the present study, the expression levels of ERBB3 in tumor and normal tissues of patients with cervical squamous cell carcinoma and cervical adenocarcinoma and in different cervical lines with or without HPV infection were measured by reverse transcription-quantitative polymerase chain reaction (RT-qPCR) and compared. Additionally, the effects of ERBB3 on cancer cell proliferation, migration and invasion were also investigated.

\section{Materials and methods}

Patients and tissue collection. A total of 25 females with cervical squamous cell carcinoma and 25 females with 
cervical adenocarcinoma who were treated at the Luodian Hospital (Shanghai, China) from July 2014 to July 2016 were enrolled in the present study. All patients were diagnosed by pathological and imaging examinations. All included patients were diagnosed with cervical cancer and were being treated for the first time. Patients with other types of malignancies (such as lung, liver, gastric cancers), other severe diseases (such as cardiovascular disease) and other cervical diseases (such as cervicitis and cervical ectropion) were excluded. The age of the patients with cervical squamous cell carcinoma ranged from 26 to 71 years, with an average age of $55 \pm 7.7$ years. The age of the patients with cervical adenocarcinoma ranged from 25 to 80 years, with an average age of $57 \pm 9.9$ years. All patients were treated with surgical resection, and tumor and normal tissues $\geq 5 \mathrm{~cm}$ around the tumor were collected during surgery. All patients provided written informed consent. The present study was approved by the ethics committee of the Luodian Hospital (Shanghai, China).

Cell lines and cell culture. Human cervical squamous cell carcinoma SiHa (HPV positive) and C33A (HPV negative) cell lines, and human normal cervical Ect1/E6E7 (HPV positive) and $\mathrm{HCvEpC}$ (HPV negative) cell lines were purchased from American Type Culture Collection (ATCC; Manassas, VA, USA). All cells were cultured in ATCC-formulated Eagle's Minimum Essential Medium (cat no. 30-2003; ATCC) containing $10 \%$ fetal bovine serum (Thermo Fisher Scientific) in an incubator $\left(37^{\circ} \mathrm{C}, 5 \% \mathrm{CO}_{2}\right)$. Cells were harvested during the logarithmic growth phase for subsequent experiments.

$R T$-qPCR. TRIzol ${ }^{\circledR}$ reagent (Invitrogen; Thermo Fisher Scientific, Inc., Waltham, MA, USA) was used to extract total RNA from the tumor tissues, adjacent healthy tissues and in vitro cultured cells of $\mathrm{SiHa}, \mathrm{C} 33 \mathrm{~A}, \mathrm{Ect1} / \mathrm{E} 6 \mathrm{E} 7$ and $\mathrm{HCvEpC}$ cell lines. Tumor and normal tissues were ground in liquid nitrogen prior to the addition of TRIzol ${ }^{\circledR}$ reagent. Following this, cDNA was then synthesized using SuperScript III Reverse Transcriptase (Thermo Fisher Scientific, Inc.) with total RNA as the template. SYBR ${ }^{\circledR}$ Green Real-Time PCR Master Mixes (Thermo Fisher Scientific, Inc.) and cDNA were then used to prepare the PCR reaction system. ERBB3 primers (cat. no. qHsaCIP0031829) were purchased from Bio-Rad Laboratories, Inc. (Hercules, CA, USA). The primers of the endogenous control $\beta$-actin were: Forward, 5'-GAC CTCTATGCCAACACAGT-3' and reverse, 5'-AGTACT TGCGCTCAGGAGGA-3'. The PCR was conducted on a CFX96 Touch $^{\mathrm{TM}}$ Real-Time PCR Detection System (Bio-Rad Laboratories, Inc.). PCR thermocycler conditions were: $95^{\circ} \mathrm{C}$ for $45 \mathrm{sec}$, followed by 40 cycles of $95^{\circ} \mathrm{C}$ for $10 \mathrm{sec}$ and $60^{\circ} \mathrm{C}$ for $45 \mathrm{sec}$, and the final extension step at $72^{\circ} \mathrm{C}$ for $5 \mathrm{~min}$. mRNA levels were quantified using the $2^{-\Delta \Delta \mathrm{Cq}}$ method (11), and the relative expression level of each gene was normalized to the endogenous control $\beta$-actin. This experiment was repeated 3 times.

Establishment of ERBB3 small interfering (si)RNA silencing cell lines. ErbB-3 siRNA (h) sc-35327 and control siRNA-A sc-370 were purchased from Santa Cruz Biotechnology, Inc. (Dallas, TX, USA). Transfection (Lipofectamine 2000 reagent (Invitrogen; Thermo Fisher Scientific, Inc.) was used to transfect $10 \mathrm{nM}$ siRNA into $5 \times 10^{5}$ cells. Cells were cultured for another $48 \mathrm{~h}$ before subsequent experiments. Cells without transfection were used as control, and cells transfected with $10 \mathrm{nM}$ control siRNA-A was used as negative control.

Western blot analysis. Total protein was extracted from cells of SiHa, C33A, Ect1/E6E7and HCvEpC cell lines using a RIPA solution (Thermo Fisher Scientific, Inc.). The BCA method was used for protein determination. A total of $30 \mu \mathrm{g}$ protein from each sample was subjected to electrophoresis using 10\% SDS-PAGE gel, followed by transfer to a polyvinylidene fluoride membrane. Following washing with TBST, membranes were incubated with 5\% skimmed milk at room temperature for $2 \mathrm{~h}$. Following washing with TBST, primary antibodies including rabbit anti-CFTR antibody $(1: 1,000$; cat. no. ab5470), rabbit anti-MTK1 antibody $(1: 2,000$; cat. no. ab186125), and rabbit anti- $\beta$-actin antibody (1:1,000; cat. no. ab8226; all Abcam, Cambridge, UK) overnight at $4^{\circ} \mathrm{C}$. Following washing with TBST, membranes were incubated with anti-rabbit IgG-HRP secondary antibody (1:1,000; cat. no. MBS435036; MyBioSource, Inc., San Diego, CA, USA) at room temperature for $2 \mathrm{~h}$. Signals were detected following the addition of ECL detection reagent (Sigma-Aldrich: Merck KGaA, Darmstadt, Germany). Image J V1.6 software (National Institutes of Health, Bethesda, MD, USA) was then used to normalize the relative expression level of each protein to endogenous control $\beta$-actin. This experiment was repeated 3 times.

Cell migration and invasion assay. The cell migratory ability was detected by Transwell cell migration assay (BD Biosciences, Franklin Lakes, NJ, USA). Briefly, $5 \times 10^{4}$ cells of SiHa and C33A cell lines in serum-free RPMI-1640 medium (Thermo Fisher Scientific, Inc.) were transferred to the upper chamber, while RPMI-1640 medium supplemented with $20 \%$ fetal calf serum (Sigma-Aldrich: Merck KGaA) was used to fill the lower chamber. The cells were incubated for $24 \mathrm{~h}$ at $37^{\circ} \mathrm{C}$, and stained with $0.5 \%$ crystal violet (Sigma-Aldrich: Merck KGaA) at room temperature for $20 \mathrm{~min}$. Stained cells were counted under an optical microscope (magnification, x20; Olympus Corporation, Tokyo, Japan). The same method was used to perform the invasion assay, with the exception that the upper chamber was pre-coated with Matrige ${ }^{\circledR}$ (EMD Millipore, Billerica, MA, USA) at room temperature for $2 \mathrm{~h}$ prior to experimentation. Cells transfected with control siRNA-A sc-370 were used as the negative control. Cells without any transfection were used as a control. This experiment was repeated 3 times. Cells were counted under a light microscope (Olympus, Japan), and cell numbers were normalized to that of control group which was set to 100 .

Cell proliferation assay. The cell proliferation assay was performed using a CCK-8 kit (Sigma-Aldrich: Merck $\mathrm{KGaA}$ ) according to manufacturer's protocol. A total of $100 \mu 1$ cell suspension containing $5 \times 10^{3}$ cells of $\mathrm{SiHa}$ and C33A cell lines were added into each well of 96-well plates, and CCK-8 solution $(10 \mu 1)$ was added into each well and were incubated for $12,24,48,72$ or $96 \mathrm{~h}$ at $37^{\circ} \mathrm{C}$. Following incubation at $37^{\circ} \mathrm{C}$ for an additional $4 \mathrm{~h}$, absorbance at $450 \mathrm{~nm}$ was measured using a microplate reader (Bio-Rad 


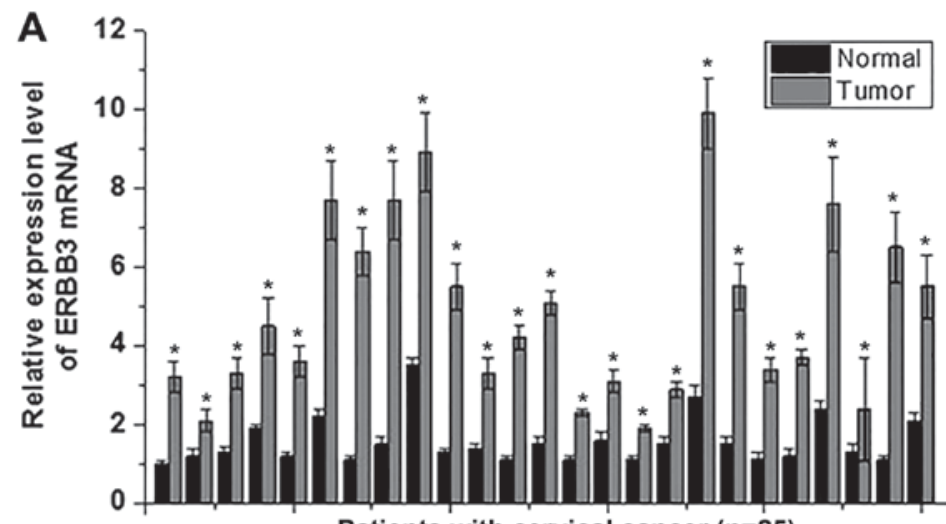

Patients with cervical cancer $(n=25)$

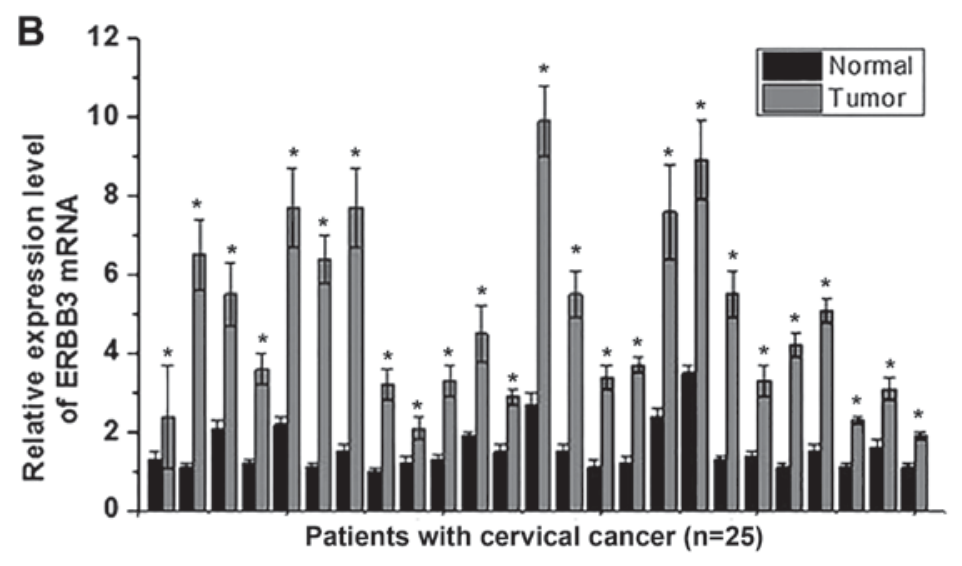

Figure 1. Expression of ERBB3 mRNA in tumor and normal tissues. (A) Relative expression level of ERBB3 mRNA in tumor and normal tissues of 25 patients with cervical squamous cell carcinoma; (B) Relative expression level of ERBB3 mRNA in tumor and normal tissues of 25 patients with cervical adenocarcinoma. ${ }^{*} \mathrm{P}<0.05$ vs. normal tissue. ERBB3, Erb-b2 receptor tyrosine kinase 3.

Laboratories, Inc.). Cells transfected with control siRNA-A sc-370 were used as the negative control. Cells without any transfection were used as a control. This experiment was repeated 3 times.

Statistical analysis. SPSS 19.0 (IBM Corp., Armonk, NY, USA) was used for all statistical analyses. Data were expressed as mean \pm standard deviation. Comparisons of data between two groups were performed using unpaired Student's t-test. Comparisons of data among multiple groups were performed using one-way analysis of variance followed by Least Significant Difference post-hoc test. $\mathrm{P}<0.05$ was considered to indicate a statistically significant difference.

\section{Results}

Expression of ERBB3 $m R N A$ in tumor and normal tissues. Expression levels of ERBB3 mRNA in tumor and normal tissues in 25 patients with cervical squamous cell carcinoma and 25 patients with cervical adenocarcinoma were detected by RT-qPCR. The results indicated that the expression level of ERBB3 mRNA was significantly higher in tumor tissue compared with normal tissue $(\mathrm{P}<0.05)$ in all 25 patients with cervical squamous cell carcinoma (Fig. 1A) and in all 25 patients with cervical adenocarcinoma (Fig. 1B). These data suggests that the downregulation of ERBB3 is likely to be involved in the pathogenesis of cervical squamous cell carcinoma and cervical adenocarcinoma.
Expression of ERBB3 protein in different cell lines. In the present study, human cervical squamous cell carcinoma $\mathrm{SiHa}$ (HPV positive) and C33A (HPV negative) cell lines and human normal cervical Ect1/E6E7 (HPV positive) and HCvEpC (HPV negative) cell lines were used. As demonstrated in Fig. 2., no significant difference in the expression level of ERBB3 protein was identified between the Ect1/E6E7 and HCvEpC cells, or between the SiHa and C33A cells, indicating that HPV infection has no significant effect on ERBB3 expression in cervical squamous cell carcinoma and normal cervical cell lines. The expression level of ERBB3 was demonstrated to be significantly increased in the $\mathrm{SiHa}$ and $\mathrm{C} 33 \mathrm{~A}$ cells compared with in the Ect1/E6E7 and HCvEpC cells, indicating an increased expression level in cervical squamous cell carcinoma. In addition, the expression level of ERBB3 was significantly decreased following siRNA silencing, indicating that the ERBB3-silenced cell lines were established successfully.

Effects of ERBB3 siRNA silencing on the proliferation of SiHa and C33A cells. As demonstrated in Fig. 3., the proliferative abilities of the SiHa and C33A cells were decreased significantly following ERBB3 siRNA silencing. These results suggest that ERBB3 expression is important for the proliferation of human cervical squamous cell carcinoma cells.

Effects of ERBB3 siRNA silencing on the migration of SiHa and C33A cells. As indicated in Fig. 4, the results of the Transwell migration assay demonstrated that the migratory abilities 
A

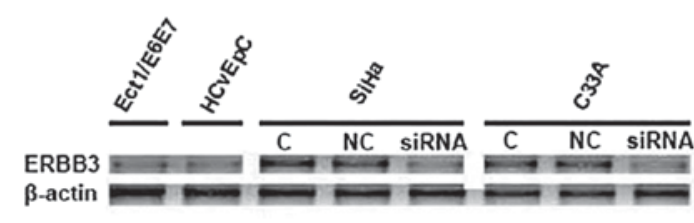

B

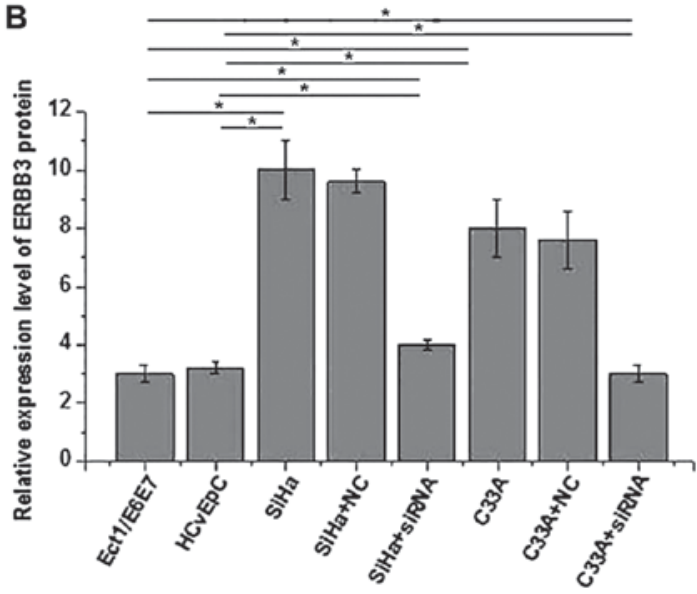

Figure 2. Expression of ERBB3 protein in different cell lines. (A) Representative results of western blot analysis; (B) Relative expression levels of ERBB3 protein in different cell lines. "P<0.05; $\mathrm{C}$, control; NC, negative control; siRNA, small interfering RNA; ERBB3, Erb-b2 receptor tyrosine kinase 3.
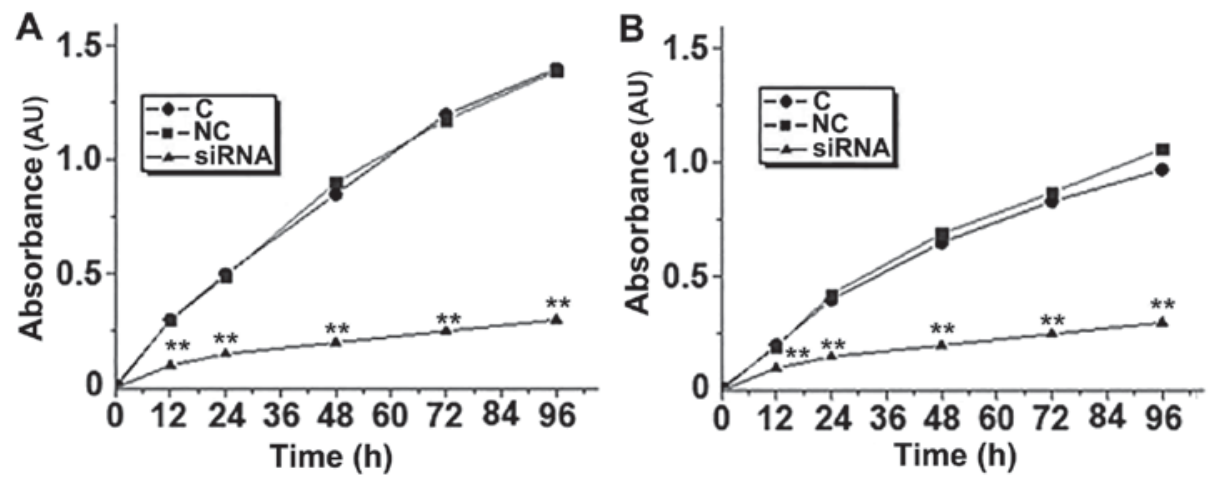

Figure 3. Cell proliferation of human cervical squamous cell carcinoma SiHa and C33A cell lines. (A) Cell proliferation of SiHa cells; (B) Cell proliferation of C33A cells. ${ }^{* *} \mathrm{P}<0.01$ vs. control cells. C, control; NC, negative control; siRNA, small interfering RNA.

of the SiHa and C33A cells were significantly decreased following EBRR3 siRNA silencing. These results suggest that EBRRS expression is important for the migration of $\mathrm{SiHa}$ and C33A cells.

Effects of ERBB3 siRNA silencing on the invasion of SiHa and C33A cells. As demonstrated in Fig. 5, the results of the invasion assay demonstrated that the invasive abilities of the $\mathrm{SiHa}$ and C33A cells were significantly decreased following EBRR3 siRNA silencing. These results suggest that the EBRRS expression is important for the invasion of SiHa and C33A cells.

Effects of ERBB3 siRNA silencing on expression of MTK-1 protein in SiHa and C33A cells. As indicated in Fig. 6, compared with $\mathrm{SiHa}$ and C33A cells without ERBB3 siRNA silencing, the expression level of the MTK-1 protein was significantly decreased in the SiHa and C33A cells with ERBB3 siRNA silencing. These results suggest that the expression level of the MTK-1 protein was decreased with the decreased expression level of ERBB3.

\section{Discussion}

As a membrane-bound protein, the functions of ERBB3 have been demonstrated to be closely associated with the occurrence and development of various human diseases, including different types of cancer $(12,13)$. In the study of breast cancer, Yan et al (12) suggested that ERBB3 served a role as an oncogene to promote the progression of breast cancer, while the miR-143/145 cluster was revealed to suppress the cell proliferation and invasion of breast cancer cells (12). The phosphorylation of ERBB3 was also demonstrated to be closely associated with the activation of the phosphoinositide 3-kinase/protein kinase B signaling pathway in the progression of a variety of types of cancer (13). In addition to its role in the pathogenesis of tumors, ERBB3 also serves a role in the formation of drug resistance developed during long-term treatment (14). Consequently, ERBB3-targeted therapy has been widely used in the treatment of cancer (15). A recent study has demonstrated that ERBB3 is likely to be involved in the occurrence of cervical cancer (8). However, the functionality of ERBB3 in cervical cancer remains unknown. The expression of ERBB3 is usually altered in tumor tissue, and it has been indicated that all ERBB family members including ERBB3 were highly expressed in ovarian carcinoma tissues, and that the increased expression level of ERBB3 may be used as an indicator in the pathological evaluation of this disease (16). In the present study, the expression level of ERBB3 was identified to be significantly increased in cervical cancer tissue compared with normal tissue in patients with cervical squamous cell carcinoma and cervical 

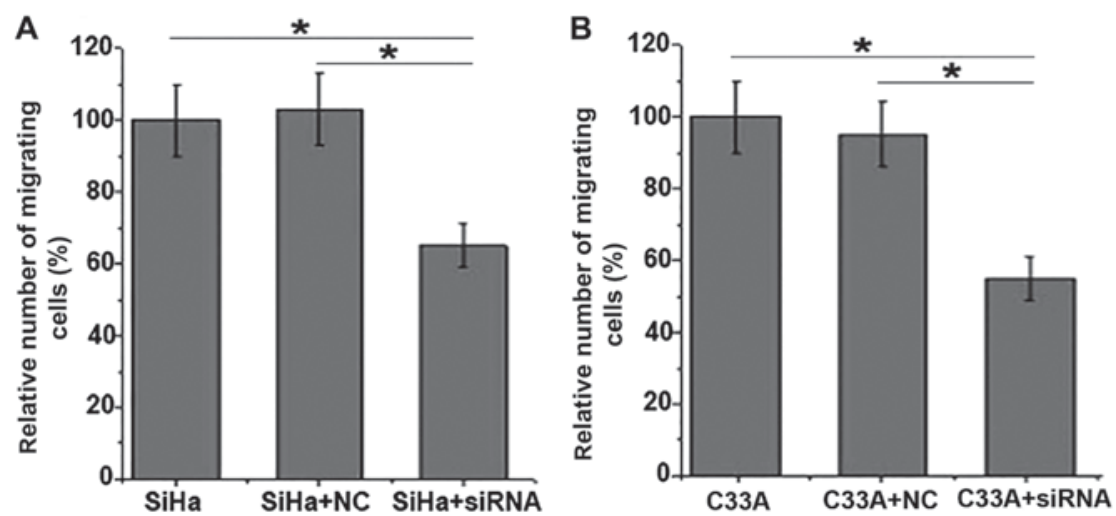

Figure 4. Relative cell migratory abilities of SiHa and C33A cells. (A) Relative cell migratory ability of SiHa cells; (B) Relative cell migratory ability of C33A cells. ${ }^{*} \mathrm{P}<0.05$. NC, negative control; siRNA, small interfering RNA.
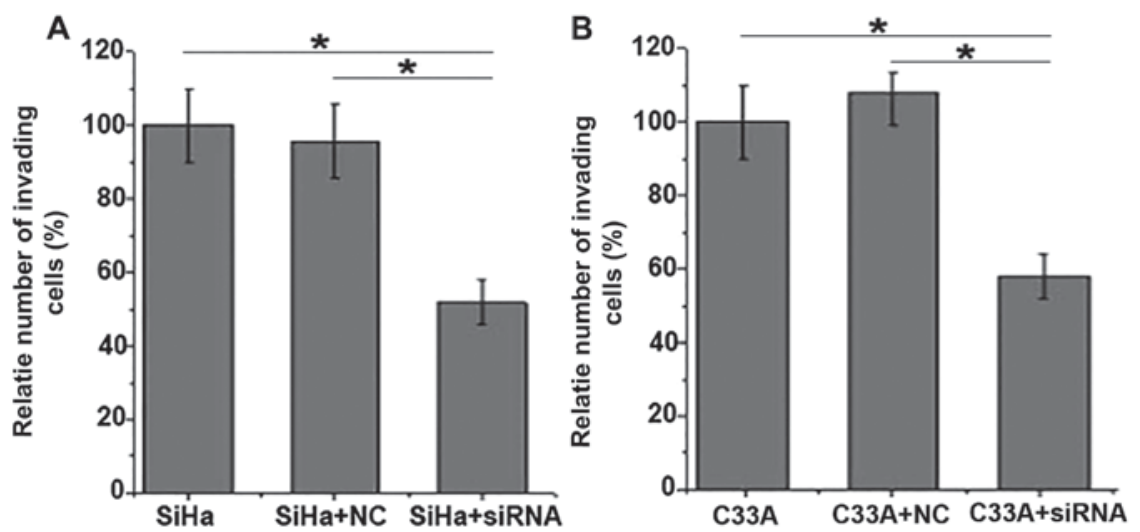

Figure 5. Relative cell invasive abilities of SiHa and C33A cells. (A) Relative cell invasive ability of SiHa cells; (B) Relative cell invasive ability of C33A cells. ${ }^{*} \mathrm{P}<0.05$. NC, negative control; siRNA, small interfering RNA.

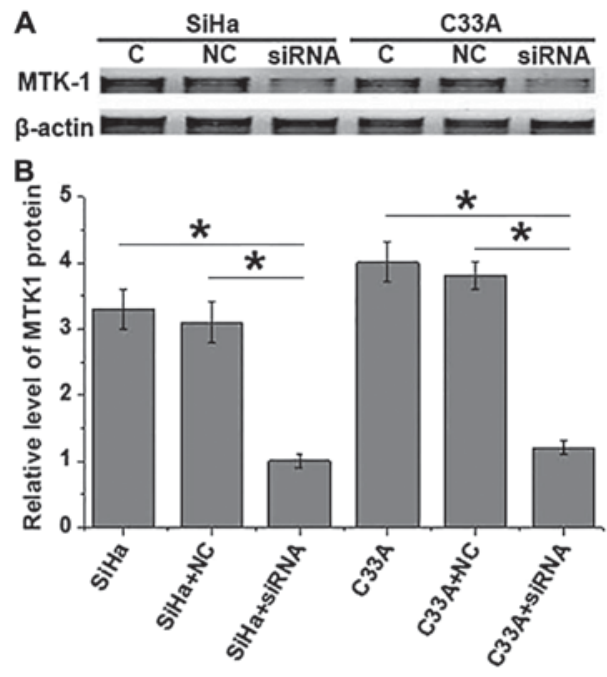

Figure 6. Expression level of MTK-1 in SiHa and C33A cell lines. (A) Representative results of western blot analysis; (B) Relative expression level of MTK-1 protein in different cell lines. "P<0.05. C, control; NC, negative control; MTK1, mitogen-activated protein kinase kinase kinase 4; siRNA, small interfering RNA.

adenocarcinoma. Furthermore, the expression level of ERBB3 was also significantly increased in cervical cancer cell lines, when compared with normal cervical cell lines. These data suggest that it is highly probable that the downregulation of ERBB3 expression is involved in the pathogenesis of cervical squamous cell carcinoma and cervical adenocarcinoma.

HPV infection is the primary contributor to the incidence of cervical cancer $(3,4)$. It is well-known that 15 out of 100 known HPV genotypes cause cervical cancer, and HPV 16 and 18 are responsible for $\sim 70 \%$ of all cervical cancer cases (17). Although HPV infection serves an essential role in the development of the majority of cervical cancers, HPV infection itself is not enough to trigger the onset of cervical cancer tumor, in which the involvement of multiple host factors, including genetic factors and environmental factors, is required (18). In the present study, no significant difference in expression level of ERBB3 was identified between the normal cervical cells with and without HPV infection and between cervical cancer cells with and without HPV infection. These data suggests that ERBB3 is not likely to be associated the HPV infection-dependent tumorigenesis of cervical cancer.

ERBB3 may participate in the development of different types of cancer by regulating the migration and invasion of tumor cells $(12,13)$. A recent study has indicated that ERBB3 may promote the development of breast cancer by increasing the migratory and invasive abilities of breast cancer cells (9), while the increased degradation of ERBB3 mediated by NRDP1 was identified to reduce the migratory and invasive abilities of human glioma cells (10). In the present study, 
ERBB3 siRNA silencing was identified to significantly reduce the proliferative, migratory and invasive abilities of cervical cancer cells, indicating that ERBB3 expression is important for the migration and invasion of cervical cancer cells. A previous study demonstrated that ERBB3 may interact with MTK1 to regulate cell migration and extracellular acidification (19). In the present study, the expression level of MTK1 protein was identified to be significantly decreased in cervical cancer cells with ERBB3 siRNA silencing compared with the cells without ERBB3 siRNA silencing, indicating an interaction between ERBB3 and MTK1 in cervical cancer cells.

In conclusion, the expression level of ERBB3 was significantly increased in cervical cancer tissue compared with normal tissue in patients with cervical squamous cell carcinoma and cervical adenocarcinoma, and ERBB3 expression was not altered by HPV infection. ERBB3 expression is important for the proliferation, migration and invasion of cervical cancer cells. The function of ERBB3 in the development of cervical cancer is likely to be achieved through the interaction with MTK1. Future studies with a greater number of patients are required to further confirm the conclusions drawn in the present study.

\section{Acknowledgements}

Not applicable.

\section{Funding}

No funding was received.

\section{Availability of data and materials}

All data generated or analyzed during this study are included in this published article.

\section{Authors' contributions}

JD and LM designed the experiments, JD and SZ performed the experiments, LW and MY analyzed the data and JD wrote the manuscript. All authors read the final manuscript.

\section{Ethics approval and consent to participate}

The present study was approved by the ethics committee of the Luodian Hospital and written informed consent was obtained from all patients.

\section{Consent for publication}

All patients provided written informed consent for publication.

\section{Competing interests}

The authors declare no that there are competing interests.

\section{References}

1. Ferlay J, Soerjomataram I, Dikshit R, Eser S, Mathers C, Rebelo M, Parkin DM, Forman D and Bray F: Cancer incidence and mortality worldwide: Sources, methods and major patterns in GLOBOCAN 2012. Int J Cancer 136: E359-E386, 2015.

2. Yang PM, Chou CJ, Tseng SH and Hung CF: Bioinformatics and in vitro experimental analyses identify the selective therapeutic potential of interferon gamma and apigenin against cervical squamous cell carcinoma and adenocarcinoma. Oncotarget 8: 46145-46162, 2017.

3. zur Hausen H: Papillomaviruses and cancer: From basic studies to clinical application. Nat Rev Cancer 2: 342-350, 2002.

4. Schiffman M, Castle PE, Jeronimo J, Rodriguez AC and Wacholder S: Human papillomavirus and cervical cancer. Lancet 370: 890-907, 2007.

5. Burd EM: Human papillomavirus and cervical cancer. Clin Microbiol Rev 16: 1-17, 2003.

6. Hildesheim A, Gonzalez P, Kreimer AR, Wacholder S, Schussler J, Rodriguez AC, Porras C, Schiffman M, Sidawy M, Schiller JT, et al: Impact of human papillomavirus (HPV) 16 and 18 vaccination on prevalent infections and rates of cervical lesions after excisional treatment. Am J Obstet Gynecol 215: 212. e1-212. e15, 2016.

7. Galic V, Herzog TJ, Lewin SN, Neugut AI, Burke WM, Lu YS, Hershman DL and Wright JD: Prognostic significance of adenocarcinoma histology in women with cervical cancer. Gynecol Oncol 125: 287-291, 2012.

8. Cancer Genome Atlas Research Network: Integrated genomic and molecular characterization of cervical cancer. Nature 543: 378-384, 2017.

9. del Pilar Camacho-Leal M, Sciortino M and Cabodi S: ErbB2 receptor in breast cancer: Implications in cancer cell migration, invasion and resistance to targeted therapy. Breast Cancer Biol Med: 2017.

10. Shi H, Gong H, Cao K, Zou S, Zhu B, Bao H, Wu Y, Gao Y, Tang Y and Yu R: Nrdp1-mediated ErbB3 degradation inhibits glioma cell migration and invasion by reducing cytoplasmic localization of p27(Kip1). J Neurooncol 124: 357-364, 2015.

11. Livak KJ and Schmittgen TD: Analysis of relative gene expression data using real-time quantitative PCR and the 2(-delta delta C(T)) method. Methods 25: 402-408, 2001.

12. Yan X, Chen X, Liang H, Deng T, Chen W, Zhang S, Liu M, Gao X, Liu Y, Zhao C, et al: miR-143 and miR-145 synergistically regulate ERBB3 to suppress cell proliferation and invasion in breast cancer. Mol Cancer 13: 220, 2014.

13. Michael N, Hopkins M and Jura N: Mechanism of PI3K activation by the HER3/ErbB3 receptor, 2016.

14. Lyu H, Huang J, Edgerton SM, Thor AD and Liu B: Abstract B20: Role of ErbB3 in tumorigenesis and drug resistance in ErbB2-driven breast cancer, 2015.

15. Ma J, Lyu H, Huang J and Liu B: Targeting of erbB3 receptor to overcome resistance in cancer treatment. Mol Cancer 13: 105, 2014.

16. Davies S, Holmes A, Lomo L, Steinkamp MP, Kang H, Muller CY and Wilson BS: High incidence of ErbB3, ErbB4 and MET expression In ovarian cancer. Int J Gynecol Pathol 33: 402, 2014.

17. Castellsagué X: Natural history and epidemiology of HPV infection and cervical cancer. Gynecol Oncol 110: S4-S7, 2008.

18. Schiffman M and Wentzensen N: Human papillomavirus infection and the multistage carcinogenesis of cervical cancer. Cancer Epidemiol Biomarkers Prev 22: 553-560, 2013.

19. Sollome JJ, Thavathiru E, Camenisch TD and Vaillancourt RR: HER2/HER3 regulates extracellular acidification and cell migration through MTK1 (MEKK4). Cell Signal 26: 70-82, 2014.

This work is licensed under a Creative Commons Attribution-NonCommercial-NoDerivatives 4.0 International (CC BY-NC-ND 4.0) License. 